\title{
Prevalence of Tuberculosis among Community Acquired Pneumonia Diagnosed Patients
}

Tinku Joseph ${ }^{1 *}$, Vinay Dharmadhikari² and Ajit Kulkarni ${ }^{2}$

${ }^{1}$ Department of Pulmonary Medicine, Amrita Institute of Medical Sciences, Kochi, Kerala, India

${ }^{2}$ Department of Pulmonary Medicine, DR. D.Y. Patil Medical college, Pimpri, Pune, India

\begin{abstract}
Aim: To evaluate the proportion of pulmonary TB among patients hospitalized with suspected communityacquired pneumonia (CAP).

Methods: Consecutive patients diagnosed with community acquired pneumonia (CAP) admitted to a tertiary care centre over a 2 year period was studied prospectively. Systematic investigation of samples of sputum and blood cultures was performed. A subset of patients had urine antigen tests and serum serology.

Results: We enrolled 104 CAP suspected patients in the study group according to the BTS guidelines for CAP. Among these patient's sputum AFB smear positivity was observed in 21 cases $(20.19 \%) .18(17.30 \%)$ patients turned out to be sputum gram stain positive, but their respective cultures didn't show any growth. $65(62.50 \%)$ of them turned out to be gram stain and culture positive for bacteriological aetiology. The Commonest cause for CAP among sputum and blood culture positive cases was Streptococcus pneumoniae (19/65) followed by, Klebsiella pneumoniae (17/65), Staphylococcus aureus (13/65), Pseudomonas aeruginosa (8/65), Escherichia coli (4/65), Acinetobactor spp. (3/65).

Conclusion: Tuberculosis is one of the leading causes of CAP in TB-prevalent areas. Our diagnostic index may help clinicians identify pulmonary TB cases immediately from CAP and initiate appropriate isolation and optimal treatment.
\end{abstract}

Keywords: Pseudomonas aeruginosa; Pulmonary TB; Pneumonia

\section{Introduction}

Tuberculosis is one of the most common infectious diseases in India known to cause significant amount of mortality and morbidity every year. Through the ages people believed that tuberculosis can present only with atypical features and only a very few studies have explained the presence of AFB positivity in CAP suspected patients presented with typical features. There have been only a few Indian reports on etiological agents for CAP. Among these most of the studies have evaluated only the presence of only common bacteria. Streptococcus Pneumoniae followed by Staphylococcal aureus, Klebsiella pneumonia and Haemophilus infleunzae still remain the commonest organisms responsible for CAP [1,2]. Although an etiological diagnosis is optimal in management of Community acquired pneumonia the responsible pathogens are not identified in $50 \%$ of patients even when extensive diagnostic tests are performed. Among this the presence of tuberculous, fungal \& viral Pneumonias in CAP patients remains grossly underdiagnosed.

Both Indian and International guidelines recommend sputum AFB smear examination after a course of antibiotics or in cases of NonResolving Pneumonias [3,4]. In Tuberculosis endemic areas, we should think of TB even in CAP suspects and they should be evaluated initially itself. This is important to cut short early transmission of infection by the undiagnosed sputum AFB positive cases. Also drugs like fluroquinolones which has got anti TB action are rampantly used in our country as an initial empiric antibiotic therapy for suspected cases of CAP, which can lead to masking of tubercular symptoms at times.

Primary aim of our study which was conducted in a tertiary referral center in Pune with a heterogeneous population representing patients from western India is to provide information concerning the prevalence of TB cases presenting as CAP. All the patients admitted as CAP suspects met the CURB 65 criteria of hospitalization according to BTS guidelines $[5,6]$.

\section{Materials and Methods}

The study comprised of 104 adult patients who were diagnosed as community-acquired pneumonia (as per BTS guideline) and admitted in in-patient ward or MICU due to their disease severity (CURB-65 $\geq$ 2 , associated co-morbidity, lack of home support etc.) were included in this study for evaluation after proper screening and ensuring that they satisfy the inclusion criteria. Institute ethics committee approval was taken prior to starting this study. Also written consent was taken from all patients.

\section{CAP Definition}

\section{As Per BTS Guidelines [4]}

- Symptoms of an acute lower respiratory tract illness (cough and at least one other lower respiratory tract symptom (e.g. dyspnoea, pleuritic pain),

- New focal chest signs on examination (e.g. bronchial breathing).

- At least one symptomatic feature (either as symptom complex of: sweating; fever; shivers; aches and pain and/or temp of $38 \mathrm{C}$ or more).

*Corresponding author: Dr.Tinku Joseph, MD, DM, FCCP. Consultant Interventional Pulmonologist, Department of Pulmonary Medicine, Amrita Institute of Medical Sciences, Kochi, Kerala, India, Tel: ;0484-2858660 E-mail: tinkujoseph2010@gmail.com

Received November 29, 2017; Accepted December 07, 2017; Published December 14, 2017

Citation: Joseph T, Dharmadhikari V, Kulkarni A (2017) Prevalence of Tuberculosis among Community Acquired Pneumonia Diagnosed Patients. J Pulm Respir Med 7: 436. doi: 10.4172/2161-105X.1000436

Copyright: @ 2017 Joseph T, et al. This is an open-access article distributed unde the terms of the Creative Commons Attribution License, which permits unrestricted use, distribution, and reproduction in any medium, provided the original author and source are credited. 
- Associated with new radiographic shadowing for which there is no other explanation

- No other explanation for the illness which is treated as CAP with antibiotics.

Patients with evidence of HIV, leukemia, lung cancer, congestive cardiac failure, evidence of tuberculosis on chest X-ray, health careassociated pneumonia and on Immunosuppressive therapy were excluded from the study. A through history was taken from the enrolled patients regarding presence of fever, cough, sputum production and pleuritic chest pain and followed by a detailed clinical examination. Complete haemogram, liver function and renal function tests, serological tests and chest $\mathrm{x}$-ray was done in all patients. ABG analysis and ECG was done when indicated.

\section{Samples collected}

Sputum collection was done at the time of admission prior to antibiotic administration in as much as cases possible. In patients who could not expectorate sputum spontaneously, sputum induction was done by nebulisation with $3 \%$ hypertonic saline, which irritates the tracheobronchial tree and produced bronchorrhoea. This was subjected to Gram smear and culture, AFB smear and BACTEC culture. Also patients in whom para-pneumonic effusion was suspected, thoracentesis was done and their respective pleural fluid samples were sent for AFB smear, Gram smear, bacterial culture, AFB culture and cytology. Fibre optic bronchoscopy (FOB) for broncho-alveolar lavage fluid when indicated was done in selected patients where adequate sputum were not obtained for investigation and bacterial isolation were not possible by other means. This also was subjected to AFB and Gram smear examination followed by bacterial and MTB BACTEC culture.

Two sets of blood culture was done in every patients included in this study. Samples for blood culture were drawn in every possible cases from two different sites (Anticubital veins of both arms in most of the cases) at least 30 mins apart.

\section{Statistical analysis}

Data was analyzed using the following statistical model Software 11 by Chi-Square and $\mathrm{Z}$ test and was represented by Pie chart and composite Bar diagram.

\section{Results}

Out of 104 CAP patients enrolled in our study group, sputum AFB smear positivity was observed in 21 cases (20.19\%). 18 (17.30\%) patients turned out to be only Gram smear positive but their respective bacterial cultures didn't show any growth. 65 (62.50\%) of them turned out to be Gram smear and culture positive for bacteriological etiology (Table 1).

In our study out of 104 CAP suspects enrolled in study, 52 patients had history of prior antibiotic treatment within 3 months. 28 patients among 65 Gram smear positive and bacterial culture Positive study group, 14 patients among 18 patients who were Gram stain positive but

\begin{tabular}{|c|c|c|}
\hline Total patients & No of cases & Percentage (\%) \\
\hline Total patients enrolled & 104 & - \\
\hline $\begin{array}{c}\text { Sputum/BAL /Blood Gram smear positive \& } \\
\text { bacterial culture Positive }\end{array}$ & 65 & 62.50 \\
\hline $\begin{array}{c}\text { Sputum/BAL/Blood Gram smear positive but no } \\
\text { growth on culture (Negative) }\end{array}$ & 18 & 17.30 \\
\hline Sputum/BAL AFB smear Positive & 21 & 20.19 \\
\hline
\end{tabular}

Table 1: Microbiological data among study population.

\begin{tabular}{|c|c|c|}
\hline Total patients & No: of cases & $\begin{array}{c}\text { Prior Antibiotic } \\
\text { treatment No: }\end{array}$ \\
\hline Total patients enrolled & 104 & 52 \\
\hline $\begin{array}{c}\text { Sputum/BAL Gram smear positive \& } \\
\text { Culture Positive }\end{array}$ & 65 & 28 \\
\hline $\begin{array}{c}\text { Sputum/BAL Gram smear positive } \\
\text { but no growth on culture (Negative) }\end{array}$ & 18 & 14 \\
\hline Sputum/BAL AFB smear Positive & 21 & 10 \\
\hline
\end{tabular}

Table 2: Prior antibiotic treatment in CAP suspects-within 3 months.

Type of antibiotic used(within 3 months)\%

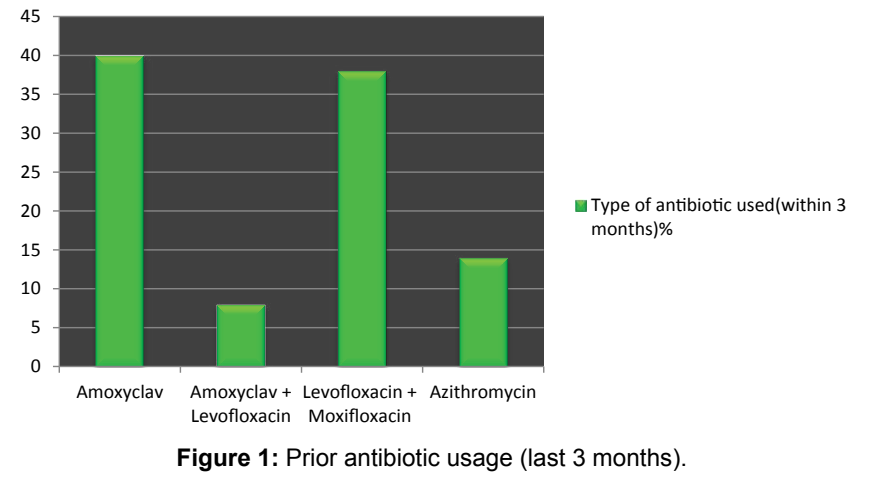

no growth on culture (Negative). 10 patients among 21 AFB positive CAP suspects had taken prior antibiotic course (Table 2).

Mainly amoxiclav, fluroquinolones like levofloxacin, moxifloxacin and macrolides like azithromycin were taken by the patients prior to admission (Figure 1).

\section{Discussion}

Tuberculosis is one of the most common infectious diseases in India, but there are only few studies which have explained the presence of AFB positivity in CAP suspected patients. Interestingly, AFB positivity was observed in our study in 21 cases (20.19\%) out of initial group of 104 patients. This study indicates that there is a high prevalence of infectious pulmonary TB in adult hospitalized CAP patients in Indian settings.

In a study conducted at Ludhiana by Gursimrat and Aggarwal $[6,7]$ regarding bacteriological etiology in CAP, they found out AFB positivity in $5 \%$ of cases. Also a similar study was done in Japan by Ishida et al. [8] regarding etiology of community acquired pneumonia in hospitalized patients which was a three year prospective study. This study also showed AFB positivity in $5.5 \%$ of the total study group.

With all these available data; clinically we should suspect pulmonary tuberculosis in patients admitted as CAP suspects, especially in TB endemic areas. Most of the CAP guidelines $[3,4]$ suggest performing sputum examination for acid- fast bacilli (AFB) for cases of nonresponders or non-resolving pneumonia. As per our observation of high incidence of pulmonary TB among CAP suspects, it's high time to strongly re-think the CAP guidelines which are currently followed, at least in Indian scenario with bulk of Tuberculosis patients presenting as CAP patients.

In our study population $38 \%$ of CAP suspects had already received a recent course of Levofloxacin and Moxifloxacin for their lower respiratory tract symptoms. It is a known fact that drugs like fluroquinolones which has got proven anti TB action are rampantly 
used in various parts as an initial empiric antibiotic therapy for suspected cases of CAP [9], which can lead to masking of tubercular symptoms at times. Indiscriminate use of antibiotics is a major problem in developing world. Resistance is also increasing to fluroquinolones, vulnerable to indiscriminate use [10]. One of the major reasons being it, that, it belongs to one of the two groups of antibiotics that are used most frequently and injudiciously as an empirical treatment for CAP and even in mild viral infections. In TB endemic zones, we should give a second thought regarding usage of these groups of antibiotics, as it can lead to drug resistance in people leading to development of MDR-TB. Also the clinical screening for Pulmonary TB among adult hospitalized CAP patients should be implemented for early diagnosis, initiation of treatment and prevention of disease spread in the community.

\section{Conclusion}

- Tuberculosis is one of the leading causes of community acquired pneumonia (CAP) in TB endemic areas.

- Our diagnostic index may help clinicians identify pulmonary TB immediately from CAP and initiate appropriate isolation and optimal treatment.

- In Indian conditions, with such a huge burden of TB, we should think of TB even in CAP suspects and should be evaluated initially itself. Both Indian and International guidelines for CAP recommend sputum AFB smear examination after course of antibiotics or in cases of non-resolving pneumonias. Time has come to give a second thought whether to follow these guidelines blindly or not. Guidelines should be modified accordingly to facilitate screening for Pulmonary TB among adult hospitalized CAP patients. This will help in early diagnosis, prompt treatment and avoid spread of infection in the community.

\section{References}

1. Kaplan V, Angus DC, Griffin MF (2002) Hospitalised community-acquired pneumonia in the elderly: Age and sex-related patterns of care and outcome in the United States. Am J Respir Crit care Med 165: 766-772.

2. Marrie TJ, Durrant H, Yastes L (1989) Community acquired pneumonia requiring hospitalization: A five year prospective study. Rev Infect Dis 11: 586-899.

3. Dheeraj G, Agarwal R, Ashutosh N, Navneet S, Narayan M, et al. (2012) Guidelines for diagnosis and management of community and hospital acquired pneumonia in adults: Joint ICS/NCCP recommendations. Lung India: 29: S27-S62.

4. Lim WS, Baudouin SV, George RC, Hill AT, Jamieson C, et al. (2009) BTS guidelines for the management of community acquired pneumonia in adults: Update 2009 Thorax: 64 Suppl 3: iii1-iii55.

5. Abhaya I, Arvind P (2005) Background papers. Burden of disease in India.

6. Gursimrat KS (2011) Tuberculosis: Current situation, challenges and overview of its control programs in India. J Glob Infect Dis 3: 143-150.

7. Aroma O, Aruna A (2006) Bacteriological profile, serology and antibiotic sensitivity pattern of micro-organisms from community acquired pneumonia. JK Science 8: 79-82.

8. Ishida T, Hashimoto T, Arta M, Ito I, Osawa M (1998) Etiology of communityacquired pneumonia in hospitalized patients. A 3 year prospective study in Japan. Chest 114: 1588-1593.

9. Grossman RF, Po-Ren H, Gillespie SH, Francesco B (2014) Communityacquired pneumonia and tuberculosis: Differential diagnosis and the use of fluoroquinolones. Int J Infectious Dis 18: 14-21.

10. Shen GH, Tsao TC, Kao SJ, Lee JJ, Chen YH, et al. (2012) Does empirical treatment of community-acquired pneumonia with fluoroquinolones delay tuberculosis treatment and result in fluoroquinolone resistance in Mycobacterium tuberculosis? Controversies and solutions. Int J Antimicrob Agents 39: 201-205. 